\title{
Design of Wireless Sweep Signal Receiving and Dispatching Hardware System Based on SiBCN Temperature Sensor
}

\author{
Huayu Wei, Tianli Lia, Li Yan, Jiyue Zhang and Xiaojie Zhang \\ Shenzhen Key Laboratory of Electromagnetic Control,Shenzhen University, Shenzhen 518060, China
}

\begin{abstract}
Based on the working principle of $\mathrm{SiBCN}$ temperature sensor and the principle of perturbation method, a wireless sweep signal transceiver system based on $\mathrm{SiBCN}$ temperature sensor covering $10 \sim 12 \mathrm{GHz}$ is designed to measure the temperature in harsh environmental such as high temperature, high oxidation and strong corrosion. Through the joint test of the system, the experimental results show that the resonant frequency and temperature of the sensor are negatively correlated at $25 \sim 350{ }^{\circ} \mathrm{C}$, that is, the temperature is increased and the frequency decreases, which is consistent with the formula.The temperature and frequency change rate is about $421.2 \mathrm{kHz} /{ }^{\circ} \mathrm{C}$, which achieve the performance indicators of the $\mathrm{SiBCN}$ temperature sensor on the hardware system.
\end{abstract}

\section{Introduction}

In industrial production, for measurements of temperature under harsh environmental conditions such as high temperature,oxidation and corrosion,ordinary temperature sensors has been unable to meet the requirements. Therefore,this paper uses a temperature-sensitive SiBCN ceramic materials based on microwave resonant wireless passive Temperature sensor [1],to measure the temperature of the harsh environment.SiBCN temperature sensor is a wireless passive microwave resonant cavity temperature sensor, belonging to Transverse Magnetic mode (TM010) resonator[2]. Based on the working principle of SiBCN temperature sensor and the principle of perturbation measurement, this paper designs a hardware system based on $\mathrm{SiBCN}$ temperature sensor wireless sweep signal transmission and reception.

\section{Sensor working principle}

The SiBCN temperature sensor is wrapped with a layer of metal platinum, which is made of $\mathrm{SiBCN}$ ceramic material.The cavity wall is filled with dielectric and connected with the slot antenna. The slot antenna is used to receive the sweep frequency signal and transmit the echo signal ,shown in Figure 1.

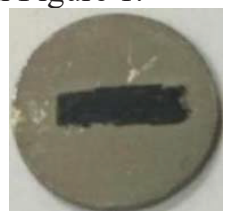

Figure 1. $\mathrm{SiBCN}$ sensor

Its working principle is the first to use the waveguide antenna transmit constant power sweep signal excitation sensor.Then, the sensor slot antenna receives the sweep signal and a certain frequency point of the sweep signal resonates with the resonant cavity.After a response period, the slot antenna sends different power amplitude echo signal received by the waveguide antenna, and the lowest amplitude point appears. When the ambient temperature of the sensor rises, the dielectric constant increases, which eventually leads to the sensor resonant frequency reduced.Finally, the lowest amplitude of the echo signal is shifted to the left.As shown in Figure 2:

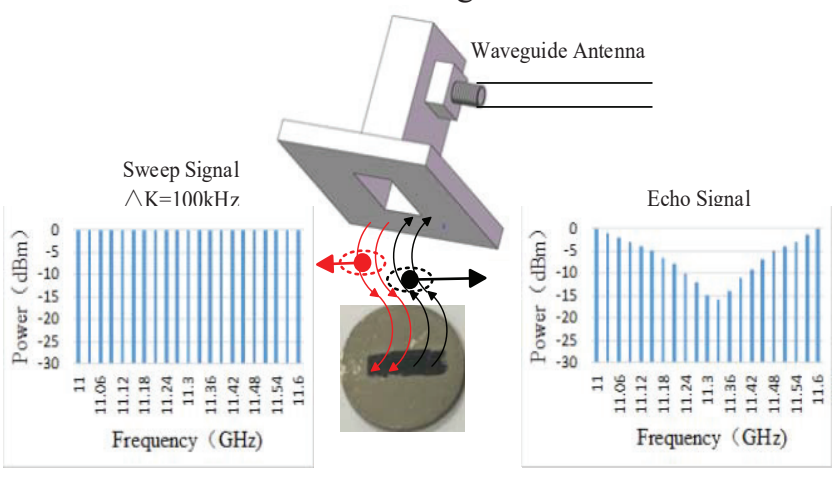

Figure 2. SiBCN sensor test schematic

The relation between the resonant frequency and the dielectric constant of a sensor is represented by a formula (1)as follows:

$$
f_{r}=\frac{P_{01}}{\pi \mathrm{a} \sqrt{u_{0} \varepsilon_{0} \varepsilon_{r}}}
$$

The fr is the resonant frequency of the resonant cavity

\footnotetext{
a Corresponding author: LTL988@gmail.com
} 
of the $\mathrm{SiBCN}$ temperature sensor,and the $\mathrm{u}_{0}$ is the vacuum permeability which is a constant.The $\varepsilon_{0}$ is the vacuum permittivity, which also known as the vacuum dielectric constant is a constant. The a is the sensor radius which is a fixed value. $P_{01}$ is the first the Bessel function of the firstkind which the first solution is a constant. And the $\varepsilon r$ is the dielectric constant of the SiBCN temperature sensor which is a variable.

With the temperature change The dielectric constant will change. The following formula shown in equation (2)

$$
\alpha_{\varepsilon}=\frac{1}{\varepsilon} \cdot \frac{d \varepsilon}{d t}
$$

The $\alpha \varepsilon$ is the temperature coefficient of the $\varepsilon$ at the $\mathrm{t}$ temperature. The $\varepsilon$ is the dielectric constant at the $t$ temperature, among them:

$$
\begin{aligned}
& d \varepsilon=\varepsilon_{r}-\varepsilon_{1} \\
& d t=t_{\mathrm{r}}-t_{1}
\end{aligned}
$$

formule(1), (2) and (3) are simplified as follows:

$$
f_{r}=\frac{P_{01}}{\pi \mathrm{a} \sqrt{u_{0} \varepsilon_{0} \cdot\left(\alpha_{\varepsilon} \varepsilon_{1} \cdot\left(t_{r}-t_{1}\right)+\varepsilon_{1}\right)}}
$$

Among (4), in addition to the tr which is variable,other parameters are known for the fixed value. It can be seen that the temperature tr and the frequency fr are inversely proportional to the relationship, that is, with the temperature increasing, the frequency decreases, the temperature decreases, the frequency increases. The different temperature values correspond to different frequencies.Using this principle, a measuring system for measuring the resonant frequency of a sensor can be designed to measure the environmental temperature.

\section{Perturbation method of measurement}

The measuring method of SiBCN temperature sensor is perturbation measurement method [4]in this paper. The principle of the perturbation method is related to the dielectric constant of the medium in the resonator of the SiBCN temperature sensor. The dielectric constant of the dielectric in the above equation (1) corresponds to the resonant frequency.

When the resonant cavity is externally excited, the excitation signal input the inner cavity of the metal SiBCN temperature sensor, reflected and superimposed. At this point, if the external excitation signal and the resonant cavity resonance occurs, it will form a standing wave in the cavity and the excitation signal energy is absorbed in the sensor.The energy reflected back at the resonant frequency point is minimum. Therefore, as long as the energy value of the echo signal is detected,the resonance of the sensor will be judged.According to this characteristic, the reflection coefficient (S11 parameter) which stand for the output signal and input signal ratio of the sensor slot antenna can be used to evaluate:

$$
S=\frac{\text { Output signal }}{\text { Input signal }}
$$

The SiBCN temperature sensor is excited by the swept signal sent by the excitation source. The slot antenna receives the swept signal and sends the echo signal.The signal frequency will not change, instead of the energy value of the signal is changed before and after stimulation. In this paper, the corresponding S11 curve is obtained by collecting the data, the ordinate of the curve represents the gain $(\mathrm{dB})$, and the abscissa represents the frequency $(\mathrm{GHz})$.

\section{System design}

Design system hardware program shown in Figure 3. The program is divided into five modules which are the controller module, the sweep signal generation module, the transmission channel conversion module, the signal conversion acquisition module and the external interface module respectively.

The controller module uses a field programmable gate array (FPGA) as a control chip. It control the HMC833 signal source to adjust the frequency range of the sweep signal, step and sweep points.It outputs the system required base frequency signal and control $\mathrm{AD}$ analog converter to collect analog signals, and converted into digital signals, data processing, drawing S11 curve and displaying. The sweep signal generating module obtains the frequency signal required for the system by double-frequency the fundamental frequency signal outputted from the signal source. The function of the transmission channel conversion module is to isolate the transceiver channel and send the sweep signal to receive the echo signal. The signal conversion acquisition module converts the power value to the voltage and collects it. The external interface module outputs data to the upper computer or the display screen.

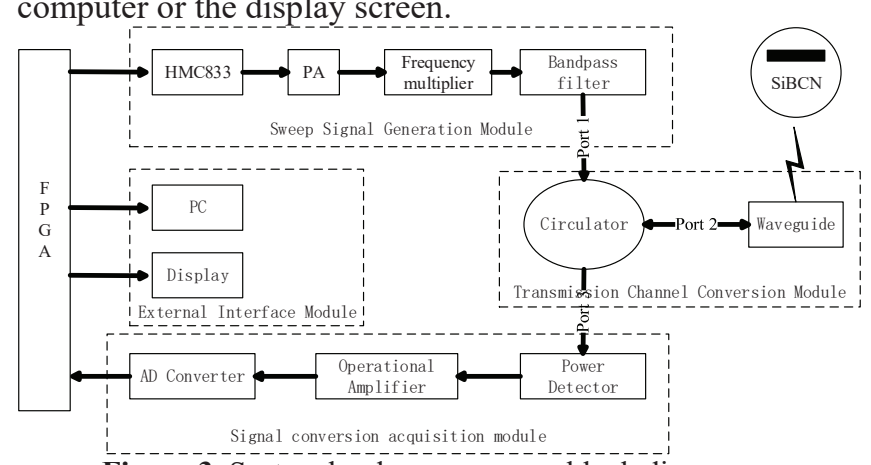

Figure 3. System hardware program block diagram

\section{Design of HMC833 Signal Source}

The signal source chip used in this system is a low phase noise $(<-170 \mathrm{dBc} / \mathrm{Hz})$ produced by Analog Devices, broadband, integrated fractional frequency divider PLL HMC833 chip [5], as shown in Figure 4.The chip is internally integrated with voltage controlled oscillator (VCO), charge pump (CP), integrated phase detector (PFD), $\Sigma-\Delta$ modulator, and $\mathrm{N}$ or $\mathrm{R}$ frequency division doubler (DIV). The VCO has a base frequency range of 0.015 to $3 \mathrm{GHz}$. The PFD and modulator give the external loop filter (LF) a wider bandwidth while suppressing external noise to improve the output signal immunity and stability. By frequency division or frequency doubling of 
the reference signal, HMC833 output frequency range reaches $0.025 \sim 6 \mathrm{GHz}$. FPGA controls HMC833 chip registers of fractional-N frequency,which can achieve 5.5 $\sim 5.8 \mathrm{GHz}$ frequency output.Then, after multiplying the frequency doubler, the frequency output of the 11.0 11.6GHz can be theoretically obtained to meet the system requirements.

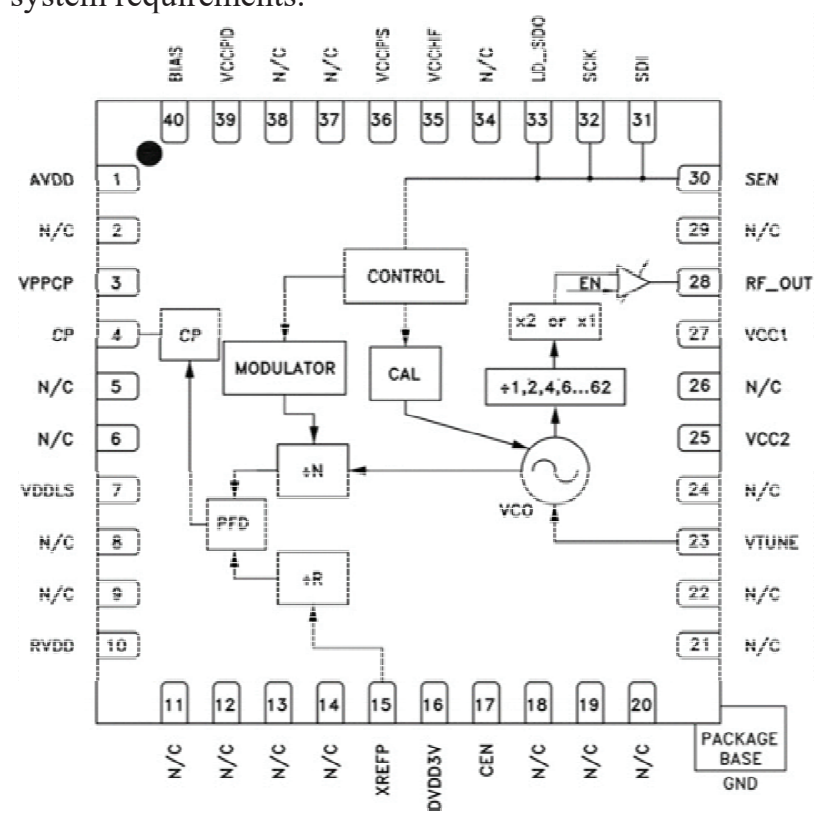

Figure 4. HMC833 internal schematic diagram

Program of frequency synthesis of HMC833 signal source is designed by PLL principle, which is called charge pump phase locked frequency synthesis. As shown in Figure 5:

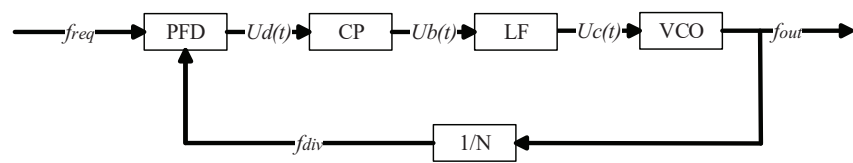

Figure 5. HMC833 signal source block diagram

The HMC833 chip integrates VCO, CP and PFD.A loop filter (LF) is designed on the periphery of the chip to form a complete PLL. Phase-locked loop operating principle[6]: firstly suppose the VCO output frequency fout $=0 \mathrm{GHz}$. Phase difference of reference frequency $\mathrm{f}_{r e q}$ and divider output frequency $\mathrm{f}_{d i v}$ is large, which makes $U_{\mathrm{d}}(t)$ maximum. Through $\mathrm{CP}$ and LF, signal of controling VCO that has been output to make the VCO oscillate with a large frequency. The frequency of the VCO output is passed through the $\mathrm{N}$ frequency division, and the frequency is compared with the reference signal frep. When there is a steady phase difference between the fdiv and the freq, the output signal that is $U_{\mathrm{d}}(t)$ stabilize. PLL implements the VCO output to follow the reference signal.

\section{System results test}

The hardware of the system is in the right of Figure 6, and the heating platform is on the left. The temperature range is at $30 \sim 350 \mathrm{C}$. The waveguide is placed the heating platforn above, and the sensor is placed at the bottom of the waveguide, as shown in Figure 6:

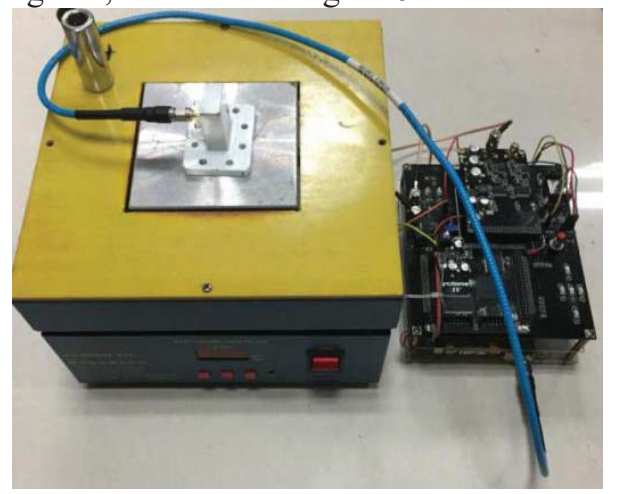

Figure 6. System hardware physical and heating platform

Figure 7 is a sensor $\mathrm{S} 11$ curve at $50^{\circ} \mathrm{C}, 100^{\circ} \mathrm{C}, 150^{\circ} \mathrm{C}$, $200^{\circ} \mathrm{C}, 250^{\circ} \mathrm{C}, 300^{\circ} \mathrm{C}$ and $350^{\circ} \mathrm{C}$, and each $\mathrm{S} 11$ curve has the lowest point. Its abscissa corresponds to the resonant frequency of sensor at the temperature and its ordinate corresponds to the S11 value of the sensor. On the whole, in the several S11 curves at $50 \sim 350^{\circ} \mathrm{C}$, the minimum value of the corresponding S11 is $-27.53 \mathrm{~dB}$, and the maximum value is $-20.8 \mathrm{~dB}$. Within the resolution range of S11 curve resonance frequency, the hardware requirements are met.

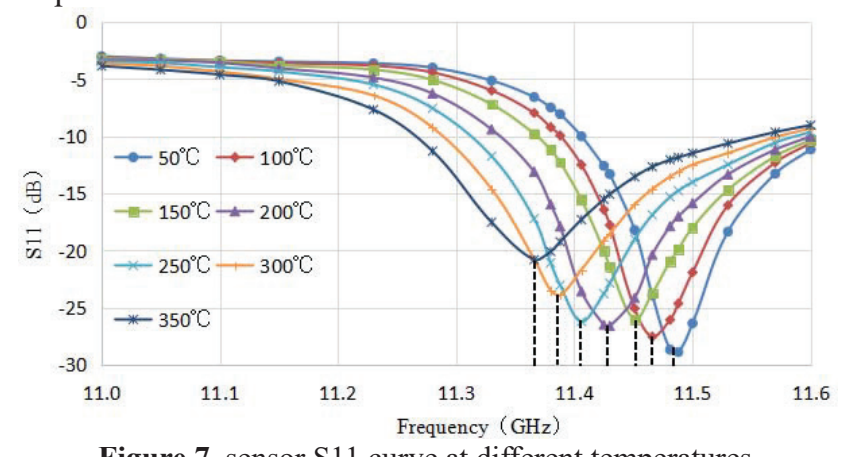

Figure 7. sensor S11 curve at different temperatures

According to the experiment, the temperature of the sensor is increased from 50 to 350 degrees by the heating platform, and the average value of the 14 data is taken every 25 degrees, as shown in figure 8 . The resonant frequencies of the sensors at 50 and 350 degrees are $11.488 \mathrm{GHz}$ and $11.360 \mathrm{GHz}$, respectively.The experimental results show that the resonant frequency decreases with the increase of the temperature of the sensor in the 50 350 degrees range, and vice versa.The experimental results accord with the theoretical deduction which is negative correlation. The temperature-frequency change rate is about $421.2 \mathrm{kHz} /{ }^{\circ} \mathrm{C}$.

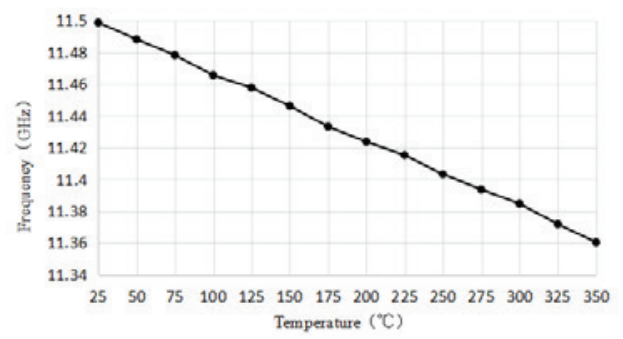

Figure 8. Temperature and resonant frequency curve 


\section{Conclusion}

Based on the principle of SiBCN temperature sensor and the principle of perturbation measurement, a hardware system of wireless frequency sweep signal receiving and transmitting based on $\mathrm{SiBCN}$ temperature sensor is designed. The system has good stability and high integration. When the temperature is $30 \sim 350^{\circ} \mathrm{C}$, the resonant frequency of the sensor is negatively correlated with the temperature, and the temperature and frequency change rate is about $421.2 \mathrm{kHz} /{ }^{\circ} \mathrm{C}$.

\section{References}

1. H. Cheng, X. Ren, S. Ebadi, et al. Wireless Passive Temperature Sensors Using Integrated Cylindrical Resonator/Antenna for Harsh-Environment Applications, IEEE Sens. J, 2015, 15(3):1453-1462.

2. Z.X. Xia, Y.J. Cheng, Y. Fan. Frequencyreconfigurable TM010-mode reentrant cylindrical cavity for microwave material processing, J. Electromagn. Waves Appl, 2013, 27(5):605-614.

3. C.P. Wang, Y.E. Liu, L.I. Ai-Xia, et al. Measurement of complex permittivity of NiZn-ferrite by resonant cavity perturbation method, Journal of Anhui University, 2012.

4. G.J. Wang. Application of the micro-disturbance method in microwave resonant-cavity analysis, Journal of Ningxia University, 2002, 23(1):50-52.

5. T. Li, Y. Chen, G. Xu, et al. Design of Low Phase Noise and Low Stray Frequency Source Based on FPGA+HMC833, International Conference on Electrical, Mechanical and Industrial Engineering. 2016.

6. W. Zhao, F. Guan, Q.I. Youguang. Implementation and Application of S-Band Frequency Synthesizer Based on Fractional N Phase-Locked Loop, Journal of Spacecraft Tt \& C Technology, 2011. 